This document is the accepted manuscript version of the following article:

Binz, T. M., Rietsche1, L., Streit, F., Hofmann, M., Gehrke, J., Herdener, M., ... Baumgartner, M. R. (2018). Endogenous cortisol in keratinized matrices: systematic determination of baseline cortisol levels in hair and the influence of sex, age and hair color. Forensic Science International, 284, 33-38.

https://doi .org/10.1016/j.forsciint.2017.12.032

This manuscript version is made available under the CC-BY-NC-ND 4.0 1icense http://creativecommons.org/1icenses/by-nc-nd/4.0/

\title{
Endogenous cortisol in keratinized matrices: systematic determination of baseline cortisol levels in hair and the influence of sex, age and hair color
}

T. M. Binz ${ }^{1}$, L. Rietschel ${ }^{2}$, F. Streit ${ }^{3}$, M. Hofmann ${ }^{4}$, J. Gehrke, M. Herdener ${ }^{5}$, B. B. Quednow $^{5}$, N. G. Martin ${ }^{6}$, M. Rietschel ${ }^{3}$, T. Kraemer $^{1}$, M. R. Baumgartner ${ }^{1}$

${ }^{1}$ Center for Forensic Hair Analytics, Zurich Institute of Forensic Medicine, University of Zurich, Zurich, Switzerland

${ }^{2} \mathrm{SRH}$ University Heidelberg, Academy for Psychotherapy, Heidelberg, Germany

${ }^{3}$ Department of Genetic Epidemiology in Psychiatry, Central Institute of Mental Health, Medical Faculty Mannheim, University of Heidelberg, Mannheim, Germany

${ }^{4}$ Swiss Federal Institute for Forest, Snow and Landscape Research, Birmensdorf, Switzerland

${ }^{5}$ Department of Psychiatry, Psychotherapy and Psychosomatics, Psychiatric Hospital, University of Zurich, Zurich, Switzerland

${ }^{6}$ Genetic Epidemiology, Queensland Institute of Medical Research, Brisbane, Australia

Keywords: endogenous hair cortisol, LC-MS/MS, sex, age, hair color

\section{Abstract}

The measurement of hair cortisol is increasingly used to measure long-term cumulative cortisol levels and investigate its role as an important stress mediator. In this study a comparative statistical analysis of five independent studies (all analyzed in our laboratory) was performed to investigate baseline ranges of cortisol values in hair and evaluate potential influences of sex, age and hair color. Cortisol concentrations in hair of 554 subjects were measured and a comparative statistical analysis was performed. The analysis showed that cortisol levels significantly differ depending on age. The toddler group ( 7 months (0.6 years) to 3 years) showed significantly higher values (median $10 \mathrm{pg} / \mathrm{mg}, p$-value $<0.0001, \mathrm{~d}=0.78$ ) 
than the adolescent group. The adolescent groups showed significantly lower ( $p$-value < $0.0001, \mathrm{~d}=0.58$ and $p<0.0001, \mathrm{~d}=0.13$ ) values (median $2.4 \mathrm{pg} / \mathrm{mg}$ and $2.8 \mathrm{pg} / \mathrm{mg}$ ) than the adult group (median $5.8 \mathrm{pg} / \mathrm{mg}$ ).

Furthermore, in the adult group men showed significantly higher cortisol values than women $(p$-value $<0.05, \mathrm{~d}=0.17)$. This effect could not be seen in the adolescent group. Black hair showed higher cortisol concentrations than blond hair ( $p$-value $<0.0001, d=1.3)$. In addition, two rounds of interlaboratory comparisons for hair cortisol samples between four laboratories revealed very consistent results.

Our results demonstrate that baseline cortisol levels are generally low in hair thus making a standardized and well-elaborated analytical method indispensable for accurate determination. Age-dependent normative baseline cortisol levels (toddlers, adolescents and adults) are highly recommended based on the comparative analysis comprising five independent studies.

\section{Introduction}

Cortisol, one of the most important glucocorticoid hormones is synthesized in the adrenal cortex and is a frequently assessed marker in psychoneuroendocrine stress research. Cortisol has been measured mainly in saliva, serum or urine in recent years. Cortisol concentration in these matrices underlies a circadian rhythm which means the concentrations fluctuate during the day. Those matrices allow the analysis of acute dynamic cortisol changes. In contrast, the effects of chronic stress and its implications on health often require the assessment of long-term cortisol levels. The analysis of cortisol in hair has therefore been a major methodological advance as it provides a retrospective measure of cumulative cortisol levels over extended time periods up to several months.

Over the past decade, the validity of hair cortisol as a measure for long term cortisol secretion has been established and the usefulness of hair cortisol as a marker for chronic 
stress has been demonstrated [1]. It has also been shown that hair cortisol is associated with a range of pathological and/or psychological conditions, such as burnout, Cushing's syndrome, and mood and anxiety disorders [2-4]. Overall, these studies show that hair cortisol has a great potential in stress research but still further research is needed on fundamental aspects that might influence hair cortisol. The establishment of standardized measurements, the definition of established values (e.g. baseline cortisol levels in untreated hair samples) and knowledge of potential confounding variables such as age, sex and hair color are important prerequisites for the establishment of hair cortisol as a biological marker in the future.

To propose established values, the quality of the analytical method used to measure hair cortisol is of great importance. Mainly immunoassay and LC-MS/MS based protocols have been used so far, revealing highly variable results $[5,6]$. Previous research has also repeatedly shown that age has an influence on hair cortisol, albeit with varying results $[5,7]$. The main research has been done on specific age groups, like young children or adults and older adults [8]. So far, there is only very limited information on hair cortisol levels in adolescents which is an age group that is very interesting due to changes in the pubertal hormone status. Previous research has also revealed highly heterogeneous findings with respect to sex differences [1]. Several studies have failed to show sex differences but newer studies imply that there are such differences [5]. The effect of natural hair color on cortisol concentration has also been studied but has mostly been found not to be correlated. Some newer literature however does show some association [9] and further investigations are needed to clarify this.

In summary, existing research has identified hair cortisol as a potential biological marker for chronic stress. However, the lack of standardized methods and inconsistent findings demonstrate the need to harmonize measurements and establish value ranges for cortisol in hair. This study was undertaken to address these gaps in research and to try to determine baseline cortisol levels in toddlers, adolescents and adults. Furthermore the influence of, sex 
and hair color on hair cortisol concentrations has been investigated to further clarify the influence of these variables and an interlaboratory comparison was performed to standardize analytical methods for cortisol measurements in hair.

\section{Material and methods}

\section{Subjects}

554 subjects from control groups of five independent studies representing the general healthy population of Switzerland and Australia were enrolled in this study. 374 adolescents were included from the "Brisbane Longitudinal Twin Study" [10, 11], 101 adults were included from the "Contact to Nature study" (Contact to nature benefits health: Mixed effectiveness of different mechanisms, submitted to International Journal of Environmental Research and Public ), 35 adults were included from the control group investigated within the "Zurich Cocaine Cognition Study" (ZuCo ${ }^{2}$ St) [12], 30 adults from the study "Neurobiological Adaptations and Pharmacological Interventions in Cocaine Addiction" (CoGlu) (clinical trials no. NCT02626494) and 14 toddlers from the Childrens' Hair Study (unpublished data). The mean age range of participants was 20.3 (range: 0.58-70, SD: 12.23). Among the participants $54 \%$ were female. The subjects declared themselves to be non-users of any drugs and provided two hair strands for analysis. Studied population was classified by sex, age and hair color. Written informed consent was provided by all participants or by a parent for underage participants.

\section{Hair sampling, extraction and LC/MS-MS analysis}

Hair samples were cut as close to the scalp as possible and in the vertex posterior region. Cortisol was analyzed in the proximal $3 \mathrm{~cm}$ hair segment. Based on an average growth rate of $1 \mathrm{~cm} /$ month this $3 \mathrm{~cm}$ segment reflects a three months' time frame prior to hair sampling. Washing and cortisol extraction followed a protocol described by Binz et al. [13] using 20 mg of hair snippets.

\section{Statistical analysis}


Shapiro Wilks Test revealed that the whole data group was not normally distributed. After log transformation data was still not normally distributed. Therefore a non-normal distribution was assumed for all the tests. All information on mean, median and standard deviation values is presented in $\mathrm{pg} / \mathrm{mg}$. Grubb's test was performed to remove outliers. From each age group one participant was removed as an outlier. Group comparisons for continuous and categorical variables (sex, hair color and age) were conducted using Kruskal-Wallis and Mann-Whitney tests. Effects sizes were transformed into Cohens'd [14]. The association between the continuous variables age and hair cortisol was assessed using Spearman correlations.

\section{Interlaboratory comparison}

The interlaboratory comparison was initiated and organized by the Center of Forensic Hair Analytics (ZFH), Switzerland. Four laboratories from Germany, Switzerland and Belgium participated in the interlaboratory comparison. The ZFH sent three previously measured authentic hair samples with low, medium and high hair cortisol concentrations to the participating labs. Each sample (sample low, sample medium and sample high) comprised $100 \mathrm{mg}$ of hair. Each of the laboratories analyzed the samples according to their standard procedures for hair cortisol analysis.

\section{Results}

Age

To investigate the influence of age on hair cortisol the study entire group was first divided into four different age groups: 7 months to 3 years (toddlers), 12 to 13 years old adolescents (adolescents 1: pre-pubertal), 14-17 years old adolescents (adolescents 2: post-pubertal) and 18 to 70 years old (adults). Kruskal-Wallis analysis revealed that there was a significant difference between the toddler group and adolescent sub-group $1(p<0.0001, d=0.9)$ and adolescent sub-group $2(p<0.0001, \mathrm{~d}=0.56)$. Adolescent group 1 showed also significant differences to the adult group $(p>0.0001, \mathrm{~d}=0.58)$ and so did adolescent group $2(p<$ $0.0001, d=0.13$ ). No significant difference could be found between adolescent group 1 and 2 . 
Therefore, both adolescents groups were combined and Kruskal-Wallis analysis revealed significant differences between the three age groups toddlers, adolescents and adults, respectively ( $p>0.0001$, Figure 1 and 2 A)). The median cortisol values in adolescents $(2.4$ $\mathrm{pg} / \mathrm{mg}$ and $2.8 \mathrm{pg} / \mathrm{mg})$ was only half as high as in adults $(5.8 \mathrm{pg} / \mathrm{mg})$ whereas the toddlers' median value was $10 \mathrm{pg} / \mathrm{mg}$ (Figure 1 and $2 \mathrm{~A}$ )). A positive Spearman correlation between participant age and hair cortisol was found in an analysis comprising the adolescents and adults (toddlers excluded) $\left(r_{s}=0.47, p<0.0001\right)$. Subgroup analysis showed that this association was present both in the adolecents $\left(r_{\mathrm{s}}=0.21, p<0.0001\right)$ and adults $\left(r_{\mathrm{s}}=0.32\right)$.
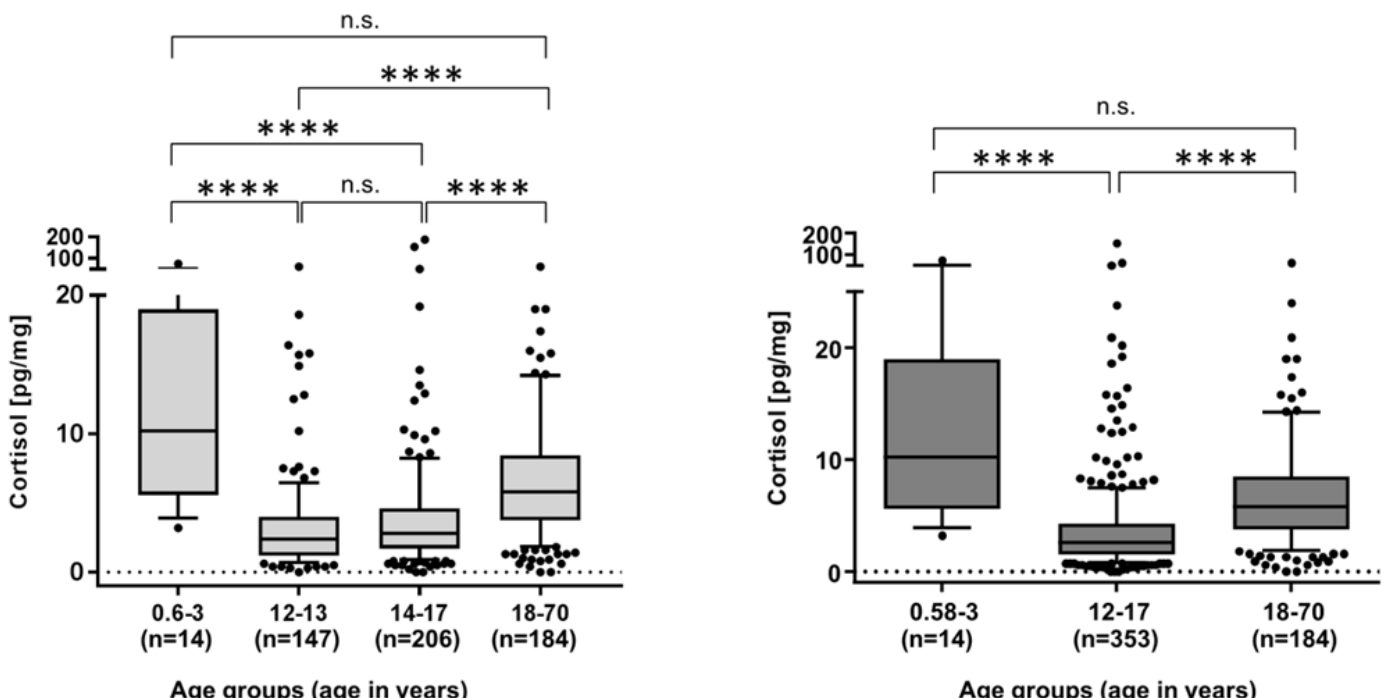

Figure 1: Comparative statistical analysis of hair cortisol concentrations in different age groups (age in years). The boxes represent the $25 \%$ and $75 \%$ percentile. The black line in the box represents the median. The whiskers represent the 10 $\%$ and $90 \%$ percentile.

A)

\begin{tabular}{ccccc}
\hline \multicolumn{5}{c}{ Cortisol [pg/mg] } \\
\hline Age groups & $\begin{array}{c}\text { (7 months } \\
\text {-3 years) }\end{array}$ & $\begin{array}{c}\text { (12-13 } \\
\text { years) }\end{array}$ & $\begin{array}{c}\text { (14-17 } \\
\text { years) }\end{array}$ & $\begin{array}{c}\text { (18-70 } \\
\text { years) }\end{array}$ \\
\hline 10\% Percentile & 3.9 & 0.7 & 0.9 & 1.9 \\
$25 \%$ Percentile & 5.6 & 1.2 & 1.7 & 3.8 \\
Median & $\mathbf{1 0}$ & $\mathbf{2 . 4}$ & $\mathbf{2 . 8}$ & $\mathbf{5 . 8}$ \\
$75 \%$ Percentile & 19 & 4.0 & 4.6 & 8.5 \\
$90 \%$ Percentile & 51 & 6.12 & 8.24 & 14 \\
\hline
\end{tabular}

B)

\begin{tabular}{|c|c|c|}
\multicolumn{4}{c}{ Toddlers } \\
$0-5 \mathrm{pg} / \mathrm{mg}$ & $5-50 \mathrm{pg} / \mathrm{mg}$ & $>50 \mathrm{pg} / \mathrm{mg}$ \\
\hline Low & Medium & High \\
\hline \multicolumn{3}{|c}{} \\
\multicolumn{3}{|c|}{ Adolescents } \\
$0-1.5 \mathrm{pg} / \mathrm{mg}$ & $1.5-7 \mathrm{pg} / \mathrm{mg}$ & $>7 \mathrm{pg} / \mathrm{mg}$ \\
\hline Low & Medium & High \\
\hline \multicolumn{3}{|c}{} \\
$0-4 \mathrm{pg} / \mathrm{mg}$ & Adults & $>15 \mathrm{pg} / \mathrm{mg}$ \\
\hline Low & $4-15 \mathrm{pg} / \mathrm{mg}$ & High \\
\hline
\end{tabular}


Figure 2: A) Median cortisol concentrations in the scalp-near $3 \mathrm{~cm}$ hair segment of the toddlers group, the adolescents' sub-groups (12-13 and 14-17 years) and adults group (18-70). B) Established hair cortisol values ranges for toddlers, adolescents and adults.

Sex

When comparing cortisol values of all participants (excluding toddlers) Mann-Whitney test revealed that male participants showed higher cortisol values than female participants $(p<$ $0.05, d=0.17$ ) (Figure 3 ). When only comparing the adolescent group no significant difference between male and female participants could be found, whereas in the adult group male adults showed higher hair cortisol levels $(p=0.026)$. In the adults group statistical analysis was performed to test if there is any effect on cortisol when comparing perimenopausal and postmenopausal women. The analysis revealed no significant changes (data not shown).
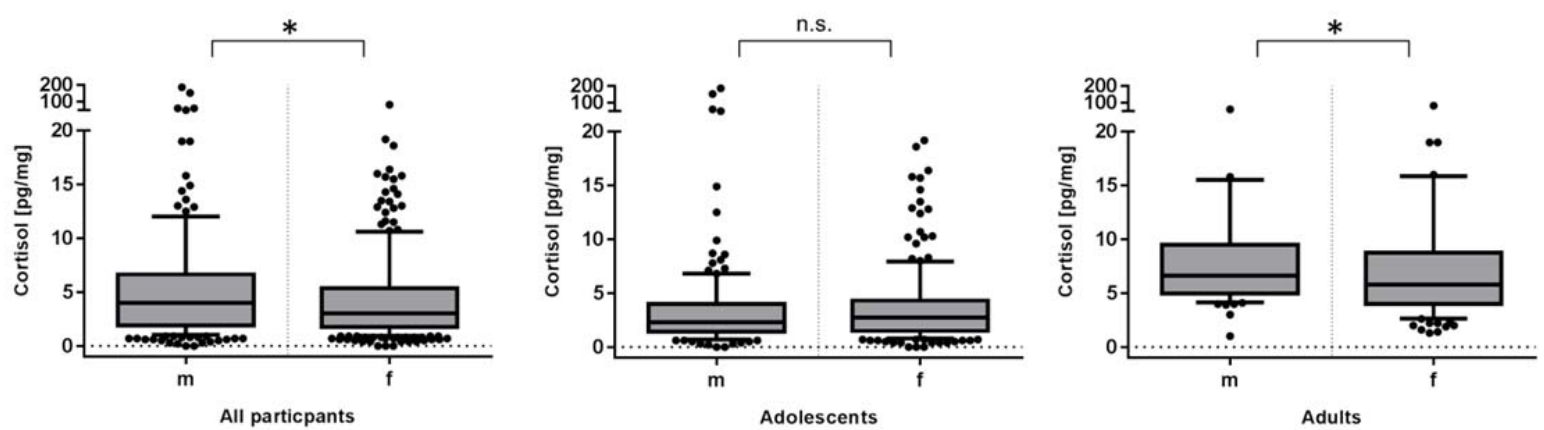

Figure 3: Comparative statistical analysis of hair cortisol concentrations in male and female subjects. The boxes represent the $25 \%$ and $75 \%$ percentile. The black line in the box represents the median. The whiskers represent the 10 $\%$ and $90 \%$ percentile.

\section{Hair color}

Hair color of all study participants (excluding toddlers) was arranged into five different groups according to darkness of hair: black, darkbrown, brown, light-brown and blond. Significant differences could be found especially between black/dark brown and blond hair $(p<0.0001$, $\mathrm{d}=1.3$ and $p<0.001, \mathrm{~d}=0.83$ ) (Figure 4). In general the median values in darker hair were 
higher than in lighter hair. The study participants from each hair color group were divided into adolescents $\left(n_{a}\right)$ and adults $\left(n_{\circ}\right)$. There were more adolescents in each group, so that an effect of age on hair color cannot be excluded. But as the adolescents group showed generally lower cortisol values, the effect of the higher values in darkbrown or brown hair is unlikely due to age. This strengthens the result of a real hair color effect.

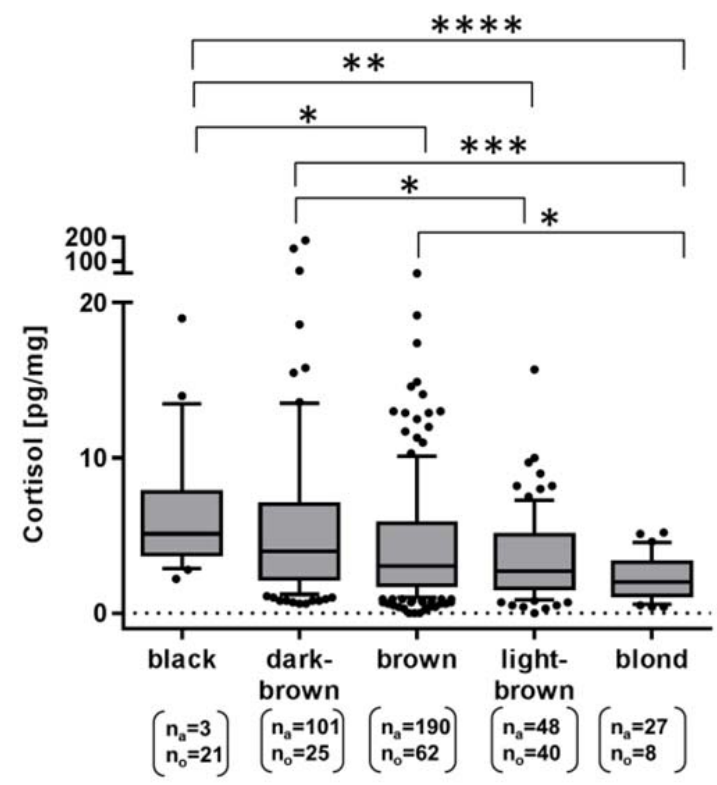

Figure 4: Comparative statistical analysis of hair cortisol concentrations depending on hair color. The boxes represent the $25 \%$ and $75 \%$ percentile. The black line in the box represents the median. The whiskers represent the $10 \%$ and 90 $\%$ percentile. $n_{a=}$ number of adolescents, $n_{0=}$ number of adults

\section{Interlaboratory comparison}

All participating laboratories analyzed the three authentic hair samples (low, medium and high) using LC-MS/MS as analytical method. Two rounds of interlaboratory comparison are presented with three participating labs each. All labs used the hair snippets as obtained; none of them pulverized the hair before analysis. Figure 5 shows the results from the measurements. In the first round, two of the three laboratories detected measureable concentration for the low sample with a median value of $0.69 \mathrm{pg} / \mathrm{mg}$ hair cortisol. One 
laboratory reported the result as negative. For the medium sample, the median value was 8.7 and for the high sample $39.8 \mathrm{pg} / \mathrm{mg}$ hair. For the second round the median value for the low sample was $0.65 \mathrm{pg} / \mathrm{mg}$, for the medium value $6.2 \mathrm{pg} / \mathrm{mg}$ and for the high sample 17.6 $\mathrm{pg} / \mathrm{mg}$.The standard deviation of all the measured results for the medium and high samples was below $30 \%$ which shows that the results were very consistent.
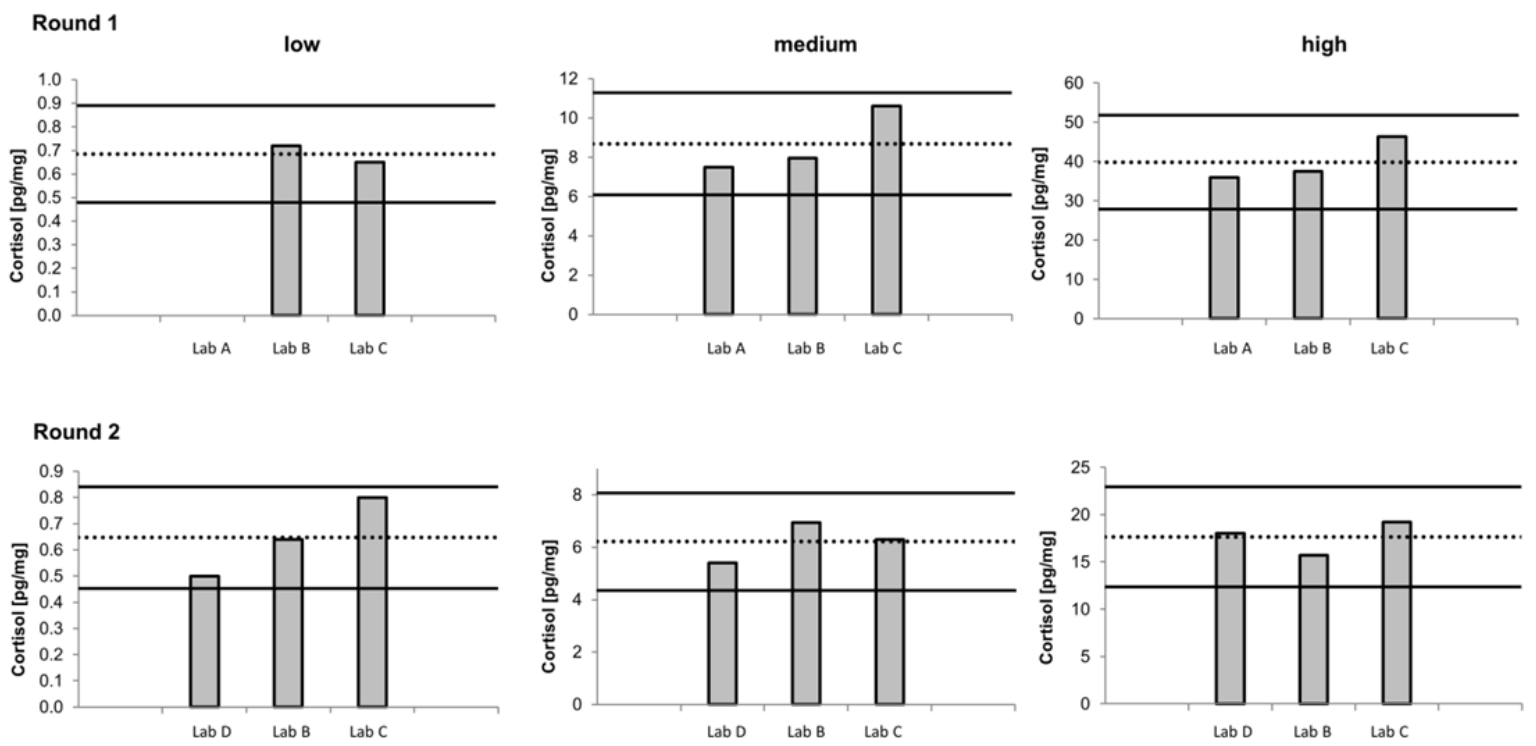

Figure 5: Two rounds of Interlaboratory comparisons between four laboratories (A,B,C, D) of hair cortisol concentrations from three authentic hair samples for each round with low, medium and high cortisol concentrations.

\section{Discussion}

In recent years, hair cortisol has emerged as a non-invasive measure for long-term hypothalamus-pituitary-adrenal (HPA) axis activity. However, previous research has been limited by the lack of well-established normative values for cortisol in adolescents and adults. Numerous studies have been performed in the past that show large deviations in hair cortisol baseline values [6]. One explanation for this fact might be the use of the analytical method. Previously, immunoassay based methods (ELISA, CLIA) have been used for determination of cortisol in hair [6]. Even though it could be shown that immunoassay and LC-MS/MS show high correlation it is known that immunoassay leads to higher results than LC-MS/MS [15]. 
The strength of our study was that we used a new, highly sensitive analytical method to measure cortisol in hair [13]. With this method, it was possible to accurately determine low endogenous cortisol concentrations in hair.

Our study suggests descriptive normative values for three specific age groups corresponding to a time period of the last three months.. Because of the non-normal distribution of the data we suggest normative ranges of hair cortisol for the age groups as depicted in Fig. 2 taken into account the $25 \%$ and $90 \%$ percentiles. These normative value ranges are based on LC-MS/MS analytics and are intended to be used as reference values for researchers using hair cortisol in research with toddlers, adolescents or adults. Nevertheless, the normative values should be used with caution and should only serve as lead values. To establish these values as real decision values (e.g. for clinical purpose to diagenose extremel high cortisol values that are linked to clinical conditions) bigger cohorts have to be analyzed.

Our results show that hair cortisol is influenced by age which has been reported before [7, 16]. Although there are studies that could not find a correlation between age and hair cortisol, a recent meta-analysis revealed that age is a covariate of hair cortisol [5]. The metaanalysis could show that hair cortisol increases with age, but also points out that hair cortisol-age correlations might be underestimated due to restricted age variance of included samples. The authors also point out that a more fine-tuned analysis for children and adolescents is needed. To our knowledge, our study is the first systematic study that investigates hair cortisol levels in a large cohort of adolescents (age group 12 to $17, n=353$ ). Most previous studies report hair cortisol concentrations for children under the age of 14 . Dettenborn et al. [7] could show that children (age: under 10) reveal higher cortisol levels than adults and especially young children show higher concentrations that decrease during their development. Our data in toddlers show the same results. Noppe et al. showed increasing hair cortisol concentrations in a study cohort of children aged 4 to 14 years [17]. Our results show that hair cortisol levels were lower in adolescents than in the adults group and support the data that cortisol concentrations increase with age. When splitting the 
adolescent group into potential prepubertal and potential postpubertal adolescents no significant changes could be seen between the groups. Of course the splitting could only be done roughly according to age (estimating that children below 14 are preburtal) but there might be children that enter puberty earlier or later. A trend can be seen though to slight higher cortisol values in the older adolescents group which goes along with the fact of increasing cortisol levels with age. Additionally higher cortisol serum levels have been described in postpubertal children [18] However the effect of steroid hormones and pubertal timing is not clear yet. Pubertal timing is a complex process that cannot be generalized to one effect. Therefore our findings are not surprising as it was described by Shi et al. previously [19]. The results are also in accordance with salivary cortisol levels that have been shown to be lower in adolescents than in adults [20]. Taken together, the results show that hair cortisol levels significantly vary from childhood to adulthood. Our data and previous literature suggest a pattern of high cortisol hair concentration in young children (toddlers) followed by low concentrations in adolescents that increase until adulthood and further in older adults. This varying pattern is probably influenced by different factors such as hair structure (different in toddlers), hormone status and/or reduced HPA axis feedback sensitivity in older age.

To further validate the hair cortisol measurements, two other factors (sex and hair color) that could influence hair cortisol concentrations were studied. It could be shown that hair cortisol was affected by sex. Tentatively, male participants showed higher cortisol levels than female participants when comparing the whole study group with the adult group only. Interestingly, no such effect could be seen for the adolescent group. Dettenborn et al. reported the same findings [7]. They found higher cortisol levels in male participants in a study group of children and adults, but not in the adolescent group. We could not conduct the analysis in the toddlers group as there were only male toddlers included in the sample group. Also the meta-analysis by Stalder et al. and a study of Staufenbiel et al. indicated that sex differences are a strong variable that influences hair cortisol $[5,21]$. However, there are studies that 
could not find such an effect $[22,23]$. Nevertheless, our study is the first large-scale study evaluating sex effects on hair cortisol in adolescents and adults, indicating that there may be an influence of male adult participants tending to have higher hair cortisol levels. A possible reason for the higher levels in men might be that men have lower corticosteroid binding globulin (CBG) levels which might result in higher free cortisol levels in men. As it is assumed that the free cortisol is incorporated in hair [24] this could explain the observed sex differences. Furthermore we analyzed if menopause could have an influence on hair cortisol. No significant differences could be observed which is in accordance with literature [25].

In addition, hair cortisol was also affected by hair color. These findings are mostly contradictory to previously reported findings when hair cortisol was mainly reported not to be affected by hair color [7, 26]. But there are also newer large studies that support our findings that darker hair color was related to higher cortisol levels $[9,21]$ and a recent study indicated that genetic variants associated with darker hair color predict higher hair cortisol concentrations [27]. It was hypothesized by other research groups that the lack of association between hair color and cortisol is explained by the fact that cortisol is incorporated independently of the melanin as it is known for acidic and neutral substances. As there are more and more studies that do see correlations of hair color and cortisol concentrations, this explanation should be reconsidered. Cortisol might bind to melanin through different kind of interaction such as ionic or hydrogen bridge bonding or through van der Waals linkage [28]. Considering literature data that show that cortisol can be washed out from hair (decline in cortisol levels from scalp-near to distal hair segments) [7, 26], it can be assumed that the binding of cortisol to melanin might not be very strong. This suggests that weaker interactions such as or hydrogen bridge bonding or van der Waals forces instead of ionic bonding are responsible for hair color differences.

To further validate the method applied in this study, we organized an interlaboratory comparison among different laboratories routinely performing hair cortisol analysis. The study clearly showed that results were very consistent and variation was below $30 \%$ when 
all laboratories analyzed the same hair samples with LC-MS/MS without further sample grinding. Russell et al. published the first and so far only interlaboratory comparison for cortisol in hair [15]. In their study hair cortisol was determined with immunoassay or LCMS/MS and the data clearly showed that the immunoassay based methods gave invariably higher results. As there were only three participating laboratories in our study, the data must be interpreted with care. Nonetheless, the study underlines the importance of the use of state-of-the-art analytical methods. Therefore, we highly recommend the use of LC-MS/MS for determination of cortisol in hair.

We are aware of the fact that the present study has limitations that have to be mentioned. First of all, the entire sample cohort was small and there are bigger cohorts available that might be considered to perform such studies (e.g. the recent meta-analysis). Nevertheless we believe that our study confirmed the results found by previous studies of similar effects on hair cortisol levels by known determinants and provides preliminary data based on a standardized analytical method that might help to establish baseline cortisol values. As already mentioned while there were variables (age, sex, hair color) that were assessed thoroughly, there were other variables that can also influence cortisol in hair and that could not be assessed in the current cohort, such as hair washing or current chronic stress [29]. Also the toddler group was very small and only male toddlers were investigated. Therefore, the present research should be further confirmed.

In conclusion, the present study evaluated age, sex and hair color as variables that can influence cortisol levels in hair and suggests ranges in hair for toddlers, adolescents and adults. The data presented should help to further establish hair cortisol in research and highlight the usefulness of the analytical method.

\section{References}


[1] L. Manenschijn, J.W. Koper, S.W. Lamberts, E.F. van Rossum, Evaluation of a method to measure long term cortisol levels, Steroids 76(10-11) (2011) 1032-6.

[2] V.L. Wester, M. Reincke, J.W. Koper, E.L. van den Akker, L. Manenschijn, C.M. Berr, J. Fazel, Y.B. de Rijke, R.A. Feelders, E.F. van Rossum, Scalp hair cortisol for diagnosis of Cushing's syndrome, Eur J Endocrinol 176(6) (2017) 695-703.

[3] S. Steudte-Schmiedgen, S. Wichmann, T. Stalder, K. Hilbert, M. Muehlhan, U. Lueken, K. BeesdoBaum, Hair cortisol concentrations and cortisol stress reactivity in generalized anxiety disorder, major depression and their comorbidity, J Psychiatr Res 84 (2017) 184-190.

[4] M. Penz, T. Stalder, R. Miller, V.M. Ludwig, M.K. Kanthak, C. Kirschbaum, Hair cortisol as a biological marker for burnout symptomatology, Psychoneuroendocrinology (2017).

[5] T. Stalder, S. Steudte-Schmiedgen, N. Alexander, T. Klucken, A. Vater, S. Wichmann, C. Kirschbaum, R. Miller, Stress-related and basic determinants of hair cortisol in humans: A metaanalysis, Psychoneuroendocrinology 77 (2017) 261-274.

[6] S.M. Staufenbiel, B.W. Penninx, A.T. Spijker, B.M. Elzinga, E.F. van Rossum, Hair cortisol, stress exposure, and mental health in humans: a systematic review, Psychoneuroendocrinology $38(8)$ (2013) 1220-35.

[7] L. Dettenborn, A. Tietze, C. Kirschbaum, T. Stalder, The assessment of cortisol in human hair: associations with sociodemographic variables and potential confounders, Stress 15(6) (2012) 578-88.

[8] S. Feller, M. Vigl, M.M. Bergmann, H. Boeing, C. Kirschbaum, T. Stalder, Predictors of hair cortisol concentrations in older adults, Psychoneuroendocrinology 39 (2014) 132-40.

[9] R.C. Rippe, G. Noppe, D.A. Windhorst, H. Tiemeier, E.F. van Rossum, V.W. Jaddoe, F.C. Verhulst, M.J. Bakermans-Kranenburg, I.M.H. van, E.L. van den Akker, Splitting hair for cortisol? Associations of socio-economic status, ethnicity, hair color, gender and other child characteristics with hair cortisol and cortisone, Psychoneuroendocrinology 66 (2016) 56-64.

[10] L. Rietschel, F. Streit, G. Zhu, K. McAloney, J. Frank, B. Couvy-Duchesne, S.H. Witt, T.M. Binz, J. McGrath, I.B. Hickie, N.K. Hansell, M.J. Wright, N. Gillespie, A.J. Forstner, T.G. Schulze, S. Wüst, M.M. Nöthen, M.R. Baumgartner, B.R. Walker, C. A.A., L. Colondro Conde, S.E. Medland, N.G. Martin, M. Rietschel, CORtisolNETwork (CORNET) Consortium, PGC MDD Group, Hair cortisol in twins: Heritability and genetic overlap with psychological variables and stress-system genes Scientific reports (2017).

[11] M.J. Wright, N.G. Martin, Brisbane Adolescent Twin Study: Outline of study methods and research projects, Australian Journal of Psychology 56(2) (2004) 65-78.

[12] M. Vonmoos, L.M. Hulka, K.H. Preller, D. Jenni, M.R. Baumgartner, R. Stohler, K.I. Bolla, B.B. Quednow, Cognitive dysfunctions in recreational and dependent cocaine users: role of attentiondeficit hyperactivity disorder, craving and early age at onset, Br J Psychiatry 203(1) (2013) 35-43.

[13] T.M. Binz, U. Braun, M.R. Baumgartner, T. Kraemer, Development of an LC-MS/MS method for the determination of endogenous cortisol in hair using (13)C3-labeled cortisol as surrogate analyte, J Chromatogr B Analyt Technol Biomed Life Sci 1033-1034 (2016) 65-72.

[14] J. Cohen, Statistical Power Analysis for the Behavioral Sciences (2nd Edition), Lawrence Erlbaum Associates, Hillsdale, 1988.

[15] E. Russell, C. Kirschbaum, M.L. Laudenslager, T. Stalder, Y. de Rijke, E.F. van Rossum, S. Van Uum, G. Koren, Toward standardization of hair cortisol measurement: results of the first international interlaboratory round robin, Ther Drug Monit 37(1) (2015) 71-5.

[16] T. Stalder, C. Kirschbaum, N. Alexander, S.R. Bornstein, W. Gao, R. Miller, S. Stark, J.A. Bosch, J.E. Fischer, Cortisol in hair and the metabolic syndrome, J Clin Endocrinol Metab 98(6) (2013) 2573-80.

[17] G. Noppe, E.F. Van Rossum, J.W. Koper, L. Manenschijn, G.J. Bruining, Y.B. de Rijke, E.L. van den Akker, Validation and reference ranges of hair cortisol measurement in healthy children, Horm Res Paediatr 82(2) (2014) 97-102.

[18] J.Y. Kang, J.Y. Park, S.I. Chun, H.S. Suh, K. Lee, R.S. Ahn, Puberty-related changes in cortisol, dehydroepiandrosterone, and estradiol-17beta secretions within the first hour after waking in premenarcheal girls, Neuroendocrinology 99(3-4) (2014) 168-77. 
[19] L. Shi, S.A. Wudy, A.E. Buyken, C. Maser-Gluth, M.F. Hartmann, T. Remer, Prepubertal glucocorticoid status and pubertal timing, J Clin Endocrinol Metab 96(6) (2011) E891-8.

[20] W. Kiess, A. Meidert, R.A. Dressendorfer, K. Schriever, U. Kessler, A. Konig, H.P. Schwarz, C.J. Strasburger, Salivary cortisol levels throughout childhood and adolescence: relation with age, pubertal stage, and weight, Pediatr Res 37(4 Pt 1) (1995) 502-6.

[21] S.M. Staufenbiel, B.W. Penninx, Y.B. de Rijke, E.L. van den Akker, E.F. van Rossum, Determinants of hair cortisol and hair cortisone concentrations in adults, Psychoneuroendocrinology 60 (2015) 182-94.

[22] W. Gao, Q. Xie, J. Jin, T. Qiao, H. Wang, L. Chen, H. Deng, Z. Lu, HPLC-FLU detection of cortisol distribution in human hair, Clin Biochem 43(7-8) (2010) 677-82.

[23] S. Fischer, R. Duncko, S.L. Hatch, A. Papadopoulos, L. Goodwin, S. Frissa, M. Hotopf, A.J. Cleare, Sociodemographic, lifestyle, and psychosocial determinants of hair cortisol in a South London community sample, Psychoneuroendocrinology 76 (2017) 144-153.

[24] S.H. Van Uum, B. Sauve, L.A. Fraser, P. Morley-Forster, T.L. Paul, G. Koren, Elevated content of cortisol in hair of patients with severe chronic pain: a novel biomarker for stress, Stress 11(6) (2008) 483-8.

[25] D. Raczkiewicz, B. Sarecka-Hujar, A. Owoc, I. Bojar, Cognitive functions and serum cortisol concentration in perimenopausal and postmenopausal women working non-manually, Neuro Endocrinol Lett 38(4) (2017) 269-274.

[26] C. Kirschbaum, A. Tietze, N. Skoluda, L. Dettenborn, Hair as a retrospective calendar of cortisol production-Increased cortisol incorporation into hair in the third trimester of pregnancy, Psychoneuroendocrinology 34(1) (2009) 32-7.

[27] A. Neumann, G. Noppe, F. Liu, M. Kayser, F.C. Verhulst, V.W.V. Jaddoe, E.F.C. van Rossum, H. Tiemeier, Predicting hair cortisol levels with hair pigmentation genes: a possible hair pigmentation bias, Sci Rep 7(1) (2017) 8529.

[28] C.R. Borges, J.C. Roberts, D.G. Wilkins, D.E. Rollins, Cocaine, benzoylecgonine, amphetamine, and $\mathrm{N}$-acetylamphetamine binding to melanin subtypes, J Anal Toxicol 27(3) (2003) 125-34.

[29] L. Dettenborn, C. Muhtz, N. Skoluda, T. Stalder, S. Steudte, K. Hinkelmann, C. Kirschbaum, C. Otte, Introducing a novel method to assess cumulative steroid concentrations: increased hair cortisol concentrations over 6 months in medicated patients with depression, Stress 15(3) (2012) 348-53. 